\title{
COVID-19 and Traumatic Brain Injury (TBI); What We Can Learn From the Viral Pandemic to Better Understand the Biology of TBI, Improve Diagnostics and Develop Evidence-Based Treatments
}

\author{
Denes V. Agoston * \\ Department of Anatomy, Physiology and Genetics, Uniformed Services University, Bethesda, MD, United States
}

Keywords: COVID-19, TBI, diseases, definitions, lessons, applications, pandemics epidemics

Traumatic brain injury (TBI) has been referred to as the "silent epidemic" but given its substantial and recurring impact on global health, it should be renamed to the "silent pandemic". The COVID19 pandemic has majorly impacted biomedical research of TBI; experimental and clinical studies have either slowed or halted and resources have been reallocated, thus resulting in a "lost year" for the TBI field. However, the pandemic can serve as an inflection point. The conceptual and technical

OPEN ACCESS

Edited by:

Mårten Risling,

Karolinska Institutet (KI), Sweden

Reviewed by:

Firas H. Kobeissy,

University of Florida, United States

*Correspondence:

Denes V. Agoston

denes.agoston@usuhs.edu

Specialty section:

This article was submitted to

Neurotrauma,

a section of the journal

Frontiers in Neurology

Received: 03 August 2021 Accepted: 01 December 2021

Published: 20 December 2021

Citation:

Agoston DV (2021) COVID-19 and Traumatic Brain Injury (TBI); What We Can Learn From the Viral Pandemic to Better Understand the Biology of TBI,

Improve Diagnostics and Develop

Evidence-Based Treatments.

Front. Neurol. 12:752937.

doi: 10.3389/fneur.2021.752937 lessons that can be learned from COVID-19 and could lead to clearer classification, improved diagnosis, and potentially improved treatment efficacy of TBI are presented in this paper.

\section{INTRODUCTION}

These are very challenging times for all mankind as severe acute respiratory syndrome coronavirus 2 (SARS-CoV-2) has been infecting the entire global population. There are already lessons learned, and even more to come after the pandemic ends, with profound effects on virtually every aspect of our lives, the economy, work, social life, and health care. Major crises, wars, and pandemics have historically forced humanity to learn, adapt, and come up with solutions by developing new technologies, business models, and even entire industries. They have also spurred major advances in biology through better understanding of diseases, identification of pathogens and their pathomechanisms, and development of novel treatments. The same is happening now during the COVID-19 pandemic, as the scientific progress is astonishing.

Within less than a year, the pathogen SARS-CoV-2 has been isolated, its genome sequenced, and the pathomechanism of coronavirus disease 2019 (COVID-19) has been identified (1, 2). Less than a year after China shared the genetic sequence of the virus (January 12, 2020) (2), an astonishing number of vaccine candidates have been in various phases of development (https://covid-19tracker.milkeninstitute.org; https://www.who.int/publications/m/ item/draft-landscape-of-covid-19-candidate-vaccines). Merely 11 months later, on December 11, 2020, the FDA authorized the first vaccine developed by BioNTech (in partnership with Pfizer) that showed $\sim 95 \%$ efficacy. Within a year of the outbreak, millions have already been vaccinated with the BioNTech/Pfizer and Moderna vaccines. This astonishingly rapid progress is due to many factors, "lessons learned" from previous epidemics, better understanding of human health and disease processes, clear understanding the pathobiology of COVID-19, identifying targets for vaccines and for pharmacological and biological treatments and critically by using a revolutionary, new mRNA-based technology. 
Furthermore, there are hundreds of treatments for COVID19 patients in various phases of clinical trials, many of which are repurposed drugs (https://covid-19tracker.milkeninstitute.org/\# treatment). Among these drugs are antivirals (e.g., Remdesivir), monoclonal antibody cocktails (e.g., REGN-COV2), and antiinflammatory drugs that are either in clinical trial or have received emergency use authorization (https://clinicaltrials.gov/ ct2/show/NCT04350593 and https://www.pnas.org/content/117/ 44/27141 and https://science.sciencemag.org/content/369/6508/ 1261 and https://www.eurekalert.org/pub_releases/2020-11/rcidnp110420.php).

While the world understandably focuses on the COVID19 pandemic, other diseases have not disappeared. TBI affects $\sim 3$ million people, kills $\sim 50,000$, and permanently disables $\sim 80,000$ people in the US alone EVERY year. (https://www.cdc. gov/traumaticbraininjury/get_the_facts.html' August 31, 2020). Worldwide numbers are roughly an order of magnitude higher and are rapidly rising especially in developing countries $(3,4)$. Based on these numbers alone, TBI should be called "silent pandemic" that occurs year after year!

This Opinion article list some of the lessons that can be learned from COVID-19 and can be apply some of them in TBI research. Some of the potential lessons are conceptual, some are technical.

\section{CONCEPTUAL}

\section{Definition}

Defining COVID-19 only as an "infection" or even a "viral infection" would be an oversimplification. Like SARS-CoV-2, the infectious agent, TBI is not the disease itself. It is only a causative event that can be either prevented or its consequences mitigated. TBI causes structural perturbance to the brain and/or damage at the molecular, cellular, and regional level of varying severity followed by pathobiological responses that determine the disease phenotype. It may seem like a matter of semantics but using the term TBI to describe the disease can lead to an overly simple thought process. Since there is no drug for treating an "infection" per se, there is and will be no drug for "treating TBI".

\section{Spectrum of TBI}

SARS-CoV-2 infection causes a spectrum of disease severities from mild, flu-like symptomatology to severe viral pneumonia (5). Despite its scariness and harrowing toll, even severe COVID-19 is a relatively simple disease phenotype compared to the disease phenotypes caused by TBI. Different types and intensities of physical forces cause different types and severities of brain injury that precipitate in varying degrees of neuropsychological/functional impairments, traditionally classified as mild, moderate, and severe. The combination of the initial physical damage and the ensuing pathobiological responses result in different disease phenotypes that are characterized by distinct dominant pathologies (6). These make TBI several orders of magnitude more complex than COVID-19. So how do we deal with such complexity? As in the case of infectious diseases where the pathogens as well as their pathomechanisms are identified, in addition to the type of primary injury, we must be able to identify the pathobiological process triggered by the initial injury.

\section{Diagnosis}

Images of people with thermal readers, the "gun-looking" devices, have become familiar scenes during COVID-19. Elevated body temperature can indicate that an individual has COVID-19 but they could also be suffering from dozens of other infectious diseases. With respect to TBI, we are currently only "checking body temperatures." Injury markers such as ubiquitin carboxyterminal hydroxylase 1 (UCH-L1), glial fibrillary acidic protein (GFAP), and neurofilament light chain (NF-L) are excellent and sensitive indicators of neuron and glia injury (7) but single post-injury time point data greatly limit their full diagnostic potential (8). They however do not provide information about the underlying pathobiological changes and cannot be used to identify disease phenotype(s). Like COVID-19 diagnostic testing, "mechanistic" biochemical markers (e.g., inflammatory, metabolic) need to be added to the current panel of damage markers so we can identify the pathobiological responses to TBI. These biomarkers will be critical for identifying disease (endo)phenotype(s) (9) and guiding specific, targeted treatments (10). However, lessons learned from COVID-19 should change how we use and interpret existing biomarker data (11). COVID19 has majorly altered the clinical trial landscape in TBI as in many other disorders and not only from the logistical and organizational perspective. The patient population in the postCOVID-19 era will be different in many ways and will present new challenges for diagnosis and prognosis in TBI.

\section{Temporal Aspect}

Clinical studies have shown that treatment with antivirals (e.g., Remdesivir) is only effective during the early phase (7-10 days) of infection $(12,13)$ while anti-inflammatory treatments (e.g., dexamethasone or anti-IL-6 monoclonals) do more harm than good during this phase $(14,15)$. Conversely, anti-inflammatory treatment can significantly reduce unfavorable outcome and death at the later phase of the disease. Inflammation is a key adaptive response to any kind of noxious stimuli in all multicellular organisms (16-18). Neuroinflammation has been extensively studied since the 1990s but all experimentally successful anti-inflammatory treatments have failed in clinical TBI trials (19). This can be plausibly explained by the lack of key information regarding the temporal pattern of the inflammatory process, and its specific components that cannot be determined by using markers of damage. We know that there is a neuroinflammatory response to TBI (20) but the temporal pattern of its different phases, reparative vs. injurious, and their cellular and humoral components are currently not well known. It is likely that the many experimentally successful pharmacological treatments, including anti-inflammatories, missed their therapeutic windows in clinical trials, an issue compounded by inter-species differences and flaws in experimental design. We know that time is a critical dimension of most disease processes, including TBI, so we must identify not only the pathobiological responses after TBI but also their temporal patterns; both are essential for developing specific 
treatments and applying them within their therapeutic window to successfully mitigate the consequences of TBI.

\section{Systemic Nature of TBI}

COVID-19 mainly involves the respiratory system but like most diseases, it is multi-systemic, it can affect all systems from gastrointestinal to the CNS $(5,21)$. Clinical and especially experimental TBI studies have almost exclusively focused on the brain, largely ignoring the rest of the body. This is based on the traditional view of the blood brain barrier (BBB) as a "brick wall" with binary function: closed or open. There is already ample evidence about the complexities of BBB function, from regulating transendothelial transport to real "opening" (22). Endothelial stress and microvascular injury are emerging as important, maybe even dominant, (endo)phenotypes across the TBI spectrum $(10,23)$. Even mild TBI can cause endothelial stress (24) and increase systemic levels of inflammatory molecules, including interleukin-6 (IL-6) (25). Many (if not most) moderate and all severe TBI patients suffer additional injuries to internal organs, muscles, and bones (i.e., polytrauma) (26). Polytrauma creates hugely complex systemic pathobiological responses (27) and damage to the cerebral vasculature that enables the cellular and humoral components of the inflammatory response to access the brain parenchyma $(20,28)$. The effect of such abnormal intracranial chemo- and cytokine milieu on the recovery process after TBI is not well understood. Importantly, this is a two-way street as illustrated by, for example, heterotopic ossification: the conversion of muscle tissue into bone which occurs in a subset of patients who also sustain TBI in addition to bone fractures (29). While the exact mechanism is unknown, soluble factors originating from the injured brain appear to alter the regenerative process and convert muscle cells into bone (30), illustrating the crosstalk between various organs affected by physical insults.

\section{Cofactor, Comorbidities}

The importance of cofactors, age, biological sex and comorbidities have been amply demonstrated in the outcomes of COVID-19 (31) and are also known in TBI (32-35). However, the overwhelming majority of experimental studies model TBI in young male rats ignoring the pediatric and elderly populations, the fastest growing segment, that together account for up to $\sim 80 \%$ of all TBI cases (34). TBI causes very different biological responses in young versus old individuals who typically have comorbidities and related medications (36-38). History of COVID-19 can change the individual's biological background with major implications from diagnosis to treatment in TBI (11, 39). Biological sex greatly impacts outcome after TBI $(32,40)$ but females are currently hugely under-represented in experimental and clinical studies despite existing knowledge about the effects of hormones on TBI-induced pathobiological responses such as inflammation (41).

\section{TECHNICAL}

\section{Big Data}

From drug and vaccine design $(42,43)$ to vaccine distribution (44), Big Data (BD), Artificial Intelligence, and Machine
Learning have been playing a critical role in the fight against the pandemic (reviewed by) (45). C3.ai (https://c3.ai/products/ c3-ai-covid-19-data-lake/) created a COVID-19 specific "data lake," a huge, analysis ready COVID-19 data repository (at no charge!), while Palantir (https://www.palantir.com/pt_media/ palantir-is-providing-coronavirus-monitoring-to-the- $\mathrm{cdc} /$ ) is providing COVID-19 monitoring, contact tracing, vaccine manufacturing and distribution. The ability to analyze huge amounts of unformatted and heterogeneous data would be especially critical for TBI (46-48). The TBI field has already made the critical first steps into BD by establishing the Federal Interagency Traumatic Brain Injury Research Informatics System (FITBIR) (https://fitbir.nih.gov; August 31,2020) using Common Data Elements (CDE) established for both preclinical and clinical TBI data $(49,50)$. Integrating FITBIR with large, biomedical data lakes that contain data from diseases other than TBI and all pathological conditions will enable better understanding of the myriad TBI-induced molecular pathologies. For instance, the cytokine IL-6 is the same molecule in COVID-19 and in TBI, with similar downstream signaling pathways and similar activators. But the importance lies is the context in which this cytokine is found. Numerous databases containing searchable data of various aspects of inflammation, anti-inflammatory molecules, molecular signaling, and disease phenotypes are available and can be queried for molecular networks using systems biology approaches $(51,52)$. BD approaches in TBI have been hampered by insufficient volumes of data and lack of data standards $(46,47,53)$, but the benefits of using BD can be transformational in identifying pharmacological and other therapies for TBI-induced disorders.

\section{Drug Repurposing}

One of the great uses of BD approaches to combat COVID-19 is in identifying drugs that target specific pathobiological processes triggered by SARS-CoV-2 infection (54). The Lancet lists ongoing clinical trials (55) and maps the evidence-based network of COVID-19 trials for the top 15 interventions. Candidate molecules include nucleotide analogs (Remdesivir) originally developed to treat Ebola (54), anti-inflammatory steroids (dexamethasone), monoclonal antibodies ( $\mathrm{mAb}$ ) (Camrelizumab and Tocilizumab, respectively) developed to treat Hodgkin's disease and rheumatoid arthritis $(56,57)$ as well as traditional Chinese medicines that activate the body's own repair and regenerative capacity (58). It has been recognized that drug repurposing is the fastest way to identify pharmacotherapies for TBI-induced pathobiologies (59). Many of the pathobiological processes induced by the injury have been identified (6) and much can be learned about the molecular targets using databases of other disorders. Many of those repurposed drugs have been successful in experimental studies but failed in clinical trials likely due to missing the therapeutic windows. Translating therapeutic windows from rodent to human is another challenge for BD (60).

\section{Novel Therapies}

mRNA-based vaccines and mAb-based immunotherapies have been game changers in the fight against COVID-19. mAb therapies have also been used to treat CNS disorders including 
multiple sclerosis and Alzheimer's Disease with varying degrees of success $(61,62)$. Altered IL-6 signaling is an important component of the TBI-induced inflammatory response (63), and existing mAbs and/or antibody cocktails targeting IL-6 signaling can be repurposed to mitigate the TBI-induced inflammatory process (56). Furthermore, a new non-inflammatory mRNA vaccine has been successful in treating experimental autoimmune encephalomyelitis (64). Autoimmunity likely plays an important role in determining long-term outcome after TBI (65-67). The new mRNA-based therapy appears to control autoreactive $\mathrm{T}$ cells without inducing systemic immune suppression (64), which is currently a major hurdle in treating autoimmune disorders. While mAb- and mRNA-based treatments can be exciting and powerful therapies in treating some of the TBI-induced pathologies, such precision medicines require similarly precise knowledge about the identity of their molecular targets and critically, their therapeutic windows.

\section{Concluding Remarks}

Experimental and clinical TBI research has generated vast amounts of data during the last several decades. Global clinical frameworks, TEAM TBI, CENTER-TBI, and now the China

\section{REFERENCES}

1. Ludwig S, Zarbock A. Coronaviruses and SARS-CoV-2: A brief overview. Anesth Analg. (2020) 131:93-6. doi: 10.1213/ANE.0000000000004845

2. Wang H, Li X, Li T, Zhang S, Wang L, Wu X, et al. The genetic sequence, origin, and diagnosis of SARS-CoV-2. Eur J Clin Microbiol Infect Dis. (2020) 39:1629-35. doi: 10.1007/s10096-020-03899-4

3. Global, regional, and national burden of traumatic brain injury and spinal cord injury, 1990-2016: a systematic analysis for the Global Burden of Disease Study 2016. Lancet Neurol. (2019) 18:56-87. doi: 10.1016/S1474-4422(18)30415-0

4. Badhiwala JH, Wilson JR, Fehlings MG. Global burden of traumatic brain and spinal cord injury. Lancet Neurol. (2019) 18:24-5. doi: 10.1016/S1474-4422(18)30444-7

5. Krishnan A, Hamilton JP, Alqahtani SA, T AW. A narrative review of coronavirus disease 2019 (COVID-19): clinical, epidemiological characteristics, and systemic manifestations. Intern Emerg Med. (2021) 1-16. doi: 10.1007/s11739-020-02616-5

6. Saatman KE, Duhaime AC, Bullock R, Maas AI, Valadka A, Manley GT. Classification of traumatic brain injury for targeted therapies. J Neurotrauma. (2008) 25:719-38. doi: 10.1089/neu.2008.0586

7. Wang KK, Yang Z, Zhu T, Shi Y, Rubenstein R, Tyndall JA, et al. An update on diagnostic and prognostic biomarkers for traumatic brain injury. Expert Rev Mol Diagn. (2018) 18:165-80. doi: 10.1080/14737159.2018.1428089

8. Bui LA, Yeboah D, Steinmeister L, Azizi S, Hier D, Wunsch D, et al. Heterogeneity in blood biomarker trajectories after mild TBI revealed by unsupervised learning. IEEE/ACM Trans Comput Biol Bioinform. (2021). doi: 10.1109/TCBB.2021.3091972

9. Hoiland RL, Robba C, Menon DK, Sekhon MS. Differential pathophysiologic phenotypes of hypoxic ischemic brain injury: considerations for post-cardiac arrest trials. Intensive Care Med. (2020). doi: 10.1007/s00134-020-06200-5

10. Sandsmark DK, Bashir A, Wellington CL, Diaz-Arrastia R. Cerebral Microvascular Injury: A Potentially Treatable Endophenotype of Traumatic Brain Injury-Induced Neurodegeneration. Neuron. (2019) 103:367-79. doi: 10.1016/j.neuron.2019.06.002

11. DeKosky ST, Kochanek PM, Valadka AB, Clark RSB, Chou SH, Au AK, et al. Blood Biomarkers for Detection of Brain Injury in COVID-19 Patients. J Neurotrauma. (2020). doi: 10.1089/neu.2020.7332 registry, provide the foundation for better understanding the biology of the disease, improving diagnostics, and developing evidence-based treatments through global collaborations. Learning from other diseases about their underlying molecular pathologies and pharmacological targets can greatly improve our understanding of TBI-induced pathobiological responses and identifying efficient treatment strategies. "Lessons learned" from COVID-19, including the importance of the temporal dimension of TBI-induced disease processes could accelerate integrating existing and novel data, guide drug repurposing and the development of novel, high-precision pharmacotherapies.

\section{AUTHOR CONTRIBUTIONS}

The author confirms being the sole contributor of this work and has approved it for publication.

\section{ACKNOWLEDGMENTS}

I want to thank Drs. David Menon and Adel Helmy for the helpful discussions and Mrs. Alaa Kamnaksh for her editorial help.

12. Zhou F, Yu T, Du R, Fan G, Liu Y, Liu Z, et al. Clinical course and risk factors for mortality of adult inpatients with COVID-19 in Wuhan, China: a retrospective cohort study. Lancet. (2020) 395:1054-62. doi: 10.1016/S0140-6736(20)30566-3

13. Wang L, Wang Y, Ye D, Liu Q. Review of the 2019 novel coronavirus (SARSCoV-2) based on current evidence. Int J Antimicrob Agents. (2020) 55:105948. doi: 10.1016/j.ijantimicag.2020.105948

14. Li H, Zhou Y, Zhang M, Wang H, Zhao Q, Liu J. Updated Approaches against SARS-CoV-2. Antimicrob Agents Chemother. (2020) 64. doi: 10.1128/AAC.00483-20

15. Ahsan W, Javed S, Bratty MA, Alhazmi HA, Najmi A. Treatment of SARSCoV-2: How far have we reached? Drug Discov Ther. (2020) 14:67-72. doi: $10.5582 /$ ddt.2020.03008

16. Medzhitov R. Origin and physiological roles of inflammation. Nature. (2008) 454:428-35. doi: 10.1038/nature07201

17. McGinn MJ, Povlishock JT. Pathophysiology of traumatic brain injury. Neurosurg Clin N Am. (2016) 27:397-407. doi: 10.1016/j.nec.2016.06.002

18. Wang A, Luan HH, Medzhitov R. An evolutionary perspective on immunometabolism. Science. (2019) 363:1-19. doi: 10.1126/science.aar3932

19. Bergold PJ. Treatment of traumatic brain injury with anti-inflammatory drugs. Exp Neurol. (2016) 275:367-80. doi: 10.1016/j.expneurol.2015.05.024

20. Needham EJ, Helmy A, Zanier ER, Jones JL, Coles AJ, Menon DK. The immunological response to traumatic brain injury. J Neuroimmunol. (2019) 332:112-25. doi: 10.1016/j.jneuroim.2019.04.005

21. Temgoua MN, Endomba FT, Nkeck JR, Kenfack GU, Tochie JN, Essouma M. Coronavirus Disease 2019 (COVID-19) as a Multi-Systemic Disease and its Impact in Low- and Middle-Income Countries (LMICs). SN Compr Clin Med. (2020) 1-11. doi: 10.1007/s42399-020-00417-7

22. Golding EM. Sequelae following traumatic brain injury. The cerebrovascular perspective. Brain Res Brain Res Rev. (2002) 38:377-88. doi: 10.1016/S0165-0173(02)00141-8

23. Jullienne A, Obenaus A, Ichkova A, Savona-Baron C, Pearce WJ, Badaut J. Chronic cerebrovascular dysfunction after traumatic brain injury. J Neurosci Res. (2016) 94:609-22. doi: 10.1002/jnr.23732

24. Major BP, McDonald SJ, O’Brien WT, Symons GF, Clough M, Costello D, et al. Serum protein biomarker findings reflective of oxidative stress and vascular abnormalities in male, but not female, collision sport athletes. Front Neurol. (2020) 11:549624. doi: 10.3389/fneur.2020.549624 
25. Simon DW, McGeachy MJ, Bayir H, Clark RS, Loane DJ, Kochanek PM. The far-reaching scope of neuroinflammation after traumatic brain injury. Nat Rev Neurol. (2017) 13:171-91. doi: 10.1038/nrneurol.2017.13

26. Risdall JE, Menon DK. Traumatic brain injury. Philos Trans R Soc Lond B Biol Sci. (2011) 366:241-50. doi: 10.1098/rstb.2010.0230

27. Guisasola MC, Alonso B, Bravo B, Vaquero J, Chana F. An overview of cytokines and heat shock response in polytraumatized patients. Cell Stress Chaperones. (2018) 23:483-9. doi: 10.1007/s12192-017-0859-9

28. Kumar RG, Diamond ML, Boles JA, Berger RP, Tisherman SA, Kochanek PM, et al. Acute CSF interleukin-6 trajectories after TBI: associations with neuroinflammation, polytrauma, and outcome. Brain Behav Immun. (2015) 45:253-62. doi: 10.1016/j.bbi.2014.12.021

29. Brady RD, Shultz SR, McDonald SJ, O’Brien TJ. Neurological heterotopic ossification: Current understanding and future directions. Bone. (2017) 109:35-42. doi: 10.1016/j.bone.2017.05.015

30. Brady RD, Grills BL, Church JE, Walsh NC, McDonald AC, Agoston DV, et al. Closed head experimental traumatic brain injury increases size and bone volume of callus in mice with concomitant tibial fracture. Sci Rep. (2016) 6:34491. doi: 10.1038/srep34491

31. Li J, Huang DQ, Zou B, Yang H, Hui WZ, Rui F, et al. Epidemiology of COVID-19: a systematic review and meta-analysis of clinical characteristics, risk factors and outcomes. J Med Virol. (2020) 93:1449-58. doi: $10.1002 / j m v .26424$

32. Slewa-Younan S, van den Berg S, Baguley IJ, Nott M, Cameron ID. Towards an understanding of sex differences in functional outcome following moderate to severe traumatic brain injury: a systematic review. J Neurol Neurosurg Psychiatry. (2008) 79:1197-201. doi: 10.1136/jnnp.2008.147983

33. Kadar R, Rochford D, Omi E, Thomas Y, Patel K, Kulstad E. Trends in demographics and outcome of patients presenting with traumatic brain injury. Clin Exp Emerg Med. (2019) 6:113-8. doi: 10.15441/ceem.18.010

34. Roozenbeek B, Maas AI, Menon DK. Changing patterns in the epidemiology of traumatic brain injury. Nat Rev Neurol. (2013) 9:231-6. doi: $10.1038 /$ nrneurol.2013.22

35. Maas A. Traumatic brain injury: Changing concepts and approaches. Chin J Traumatol. (2016) 19:3-6. doi: 10.1016/j.cjtee.2016.01.001

36. Prins ML, Lee SM, Cheng CL, Becker DP, Hovda DA. Fluid percussion brain injury in the developing and adult rat: a comparative study of mortality, morphology, intracranial pressure and mean arterial blood pressure. Brain Res Dev Brain Res. (1996) 95:272-82. doi: 10.1016/0165-3806(96)00098-3

37. Menon DK, Ercole A. Critical care management of traumatic brain injury. Handb Clin Neurol. (2017) 140:239-74. doi: 10.1016/B978-0-444-63600-3.00014-3

38. Stocchetti N, Carbonara M, Citerio G, Ercole A, Skrifvars MB, Smielewski P, et al. Severe traumatic brain injury: targeted management in the intensive care unit. Lancet Neurol. (2017) 16:452-64. doi: 10.1016/S1474-4422(17)30118-7

39. Needham EJ, Chou SH, Coles AJ, Menon DK. Neurological Implications of COVID-19 Infections. Neurocrit Care. (2020) 32:667-71. doi: 10.1007/s12028-020-00978-4

40. Kim T, Chelluboina B, Chokkalla AK, Vemuganti R. Age and sex differences in the pathophysiology of acute CNS injury. Neurochem Int. (2019) 127:22-8. doi: 10.1016/j.neuint.2019.01.012

41. Gupte R, Brooks W, Vukas R, Pierce J, Harris J. Sex differences in traumatic brain injury: what we know and what we should know. J Neurotrauma. (2019) 36:3063-91. doi: 10.1089/neu.2018.6171

42. Panda PK, Arul MN, Patel P, Verma SK, Luo W, Rubahn HG, et al. Structure-based drug designing and immunoinformatics approach for SARS-CoV-2. Sci Adv. (2020) 6:eabb8097. doi: 10.1126/sciadv.ab b8097

43. Zhang Y, Kutateladze TG. Molecular structure analyses suggest strategies to therapeutically target SARS-CoV-2. Nat Commun. (2020) 11:2920. doi: 10.1038/s41467-020-16779-4

44. Chang S, Pierson E, Koh PW, Gerardin J, Redbird B, Grusky D, et al. Mobility network models of COVID-19 explain inequities and inform reopening. Nature. (2021) 589:82-7. doi: 10.1038/s41586-020-2923-3

45. Mbunge E, Akinnuwesi B, Fashoto SG, Metfula AS, Mashwama P, A. critical review of emerging technologies for tackling COVID-19 pandemic. Hum Behav Emerg Technol. (2020). doi: 10.1002/hbe2.237
46. Huie JR, Mondello S, Lindsell CJ, Antiga L, Yuh EL, Zanier ER, et al. Biomarkers for traumatic brain injury: data standards and statistical considerations. J Neurotrauma. (2020).

47. Hawkins BE, Huie JR, Almeida C, Chen J, Ferguson AR. Data dissemination: shortening the long tail of traumatic brain injury dark data. J Neurotrauma. (2019). doi: 10.1089/neu.2018.6192

48. Agoston DV. Big Data, Artificial Intelligence and Machine Learning in Neurotrauma. In: Kobeissy FAA, Zaraket FA, Wang K, editor. Leveraging Biomedical and Healthcare Data: Semantics, Analytics and Knowledge. London: Academic Press. (2019). p. 53-75. doi: 10.1016/B978-0-12-809556-0.00004-6

49. Thompson HJ, Vavilala MS, Rivara FP. Chapter 1 common data elements and federal interagency traumatic brain injury research informatics system for TBI research. Annu Rev Nurs Res. (2015) 33:1-11. doi: 10.1891/0739-6686.33.1

50. LaPlaca MC, Huie JR, Alam HB, Bachstetter AD, Bayir H, Bellgowan PF, et al. Pre-clinical common data elements for traumatic brain injury research: progress and use cases. J Neurotrauma. (2021). doi: 10.1089/neu.2020.7328

51. Mutowo P, Bento AP, Dedman N, Gaulton A, Hersey A, Lomax J, et al. A drug target slim: using gene ontology and gene ontology annotations to navigate protein-ligand target space in ChEMBL. J Biomed Semantics. (2016) 7:59. doi: 10.1186/s13326-016-0102-0

52. Wang K, Xiao J, Liu X, Jiang Z, Zhan Y, Yin $T$, et al. AICD: an integrated anti-inflammatory compounds database for drug discovery. Sci Rep. (2019) 9:7737. doi: 10.1038/s41598-019-4 4227-x

53. Huie JR, Almeida CA, Ferguson AR. Neurotrauma as a big-data problem. Curr Opin Neurol. (2018) 31:702-8. doi: 10.1097/WCO.00000000000 00614

54. Gordon DE, Jang GM, Bouhaddou M, Xu J, Obernier K, O’Meara MJ, et al. A SARS-CoV-2-human protein-protein interaction map reveals drug targets and potential drug-repurposing. bioRxiv. (2020).

55. Thorlund K, Dron L, Park J, Hsu G, Forrest JI, Mills EJ, et al. real-time dashboard of clinical trials for COVID-19. Lancet Digit Health. (2020) 2:e286-e7. doi: 10.1016/S2589-7500(20)3 0086-8

56. Harrison C. Focus shifts to antibody cocktails for COVID-19 cytokine storm. Nat Biotechnol. (2020) 38:905-8. doi: 10.1038/s41587-020-0634-9

57. Abbasi J. COVID-19 antibody trials have begun. Jama. (2020) 324:128 doi: $10.1001 /$ jama.2020.11582

58. Cyranoski D. China is promoting coronavirus treatments based on unproven traditional medicines. Nature. (2020). doi: 10.1038/d41586-020-01284-X

59. Gasparini F, Di Paolo T. Drug repurposing: Old drugs, new tricks to fast track drug development for the brain. Neuropharmacology. (2019) 147:1-3. doi: 10.1016/j.neuropharm.2019.01.009

60. Agoston DV, Vink B, Helmy A, Risling M, Nelson DW, Prins MPD. How to translate time; the temporal aspects of rodent and human pathobiological processes in traumatic brain injury. J Neurotrauma. (2019). doi: 10.1089/neu.2018.6261

61. Wootla B, Watzlawik JO, Stavropoulos N, Wittenberg NJ, Dasari H, Abdelrahim MA, et al. Recent advances in monoclonal antibody therapies for multiple sclerosis. Expert Opin Biol Ther. (2016) 16:827-39. doi: 10.1517/14712598.2016.11 58809

62. Alpaugh M, Cicchetti F, A. brief history of antibody-based therapy. Neurobiol Dis. (2019) 130:104504. doi: 10.1016/j.nbd.2019.104504

63. Choy EH, De Benedetti F, Takeuchi T, Hashizume M, John MR, Kishimoto T. Translating IL-6 biology into effective treatments. Nat Rev Rheumatol. (2020) 16:335-45. doi: 10.1038/s41584-020-0419-Z

64. Krienke C, Kolb L, Diken E, Streuber M, Kirchhoff S, Bukur T, et al. A noninflammatory mRNA vaccine for treatment of experimental autoimmune encephalomyelitis. Science. (2021) 371:145-53. doi: 10.1126/science.aa y3638

65. Wang KK, Yang Z, Yue JK, Zhang Z, Winkler EA, Puccio AM, et al. Plasma anti-glial fibrillary acidic protein autoantibody levels during the acute and chronic phases of traumatic brain injury: a transforming research and clinical knowledge in traumatic brain injury pilot study. J Neurotrauma. (2016) 33:1270-7. doi: 10.1089/neu.2015.3881 
66. Zhang Z, Zoltewicz JS, Mondello S, Newsom KJ, Yang Z, Yang $B$, et al. Human traumatic brain injury induces autoantibody response against glial fibrillary acidic protein and its breakdown products. PLoS ONE. (2014) 9:e92698. doi: 10.1371/journal.pone.00 92698

67. Attilio PJ, Flora M, Kamnaksh A, Bradshaw DJ, Agoston D, Mueller GP. The effects of blast exposure on protein deimination in the brain. Oxid Med Cell Longev. (2017) 2017:8398072. doi: 10.1155/2017/83 98072

Conflict of Interest: The author declares that the research was conducted in the absence of any commercial or financial relationships that could be construed as a potential conflict of interest.
Publisher's Note: All claims expressed in this article are solely those of the authors and do not necessarily represent those of their affiliated organizations, or those of the publisher, the editors and the reviewers. Any product that may be evaluated in this article, or claim that may be made by its manufacturer, is not guaranteed or endorsed by the publisher.

Copyright (c) 2021 Agoston. This is an open-access article distributed under the terms of the Creative Commons Attribution License (CC BY). The use, distribution or reproduction in other forums is permitted, provided the original author(s) and the copyright owner(s) are credited and that the original publication in this journal is cited, in accordance with accepted academic practice. No use, distribution or reproduction is permitted which does not comply with these terms. 